\title{
Research Paper: \\ Accuracy of Niacin Skin Flush Test for Diagnosing Schizophrenia
}

${ }^{*}$ Mohammad Arzanlou ${ }^{1} \odot$, Alireza Armanikian² ${ }^{\circ}$, Omid Saed $^{3}$, Faramarz Dobakhti ${ }^{4}$, Nima Motamed ${ }^{\circ}$, Akefeh Ahmadiafshar $^{6}$ (1)

1. Department of Psychiatry, School of Medicine, Zanjan University of Medical Sciences, Zanjan, Iran.

2. Department of Psychiatry, Shahid Beheshti Hospital, Zanjan University of Medical Sciences, Zanjan, Iran.

3. Department of Clinical Psychology, School of Medicine, Zanjan University of Medical Sciences, Zanjan, Iran

4. Department of Pharmaceutics, School of Pharmacy, Zanjan University of Medical Sciences, Zanjan, Iran.

5. Department of Community Medicine, School of Medicine, Zanjan University of Medical Sciences, Zanjan, Iran.

6. Department of Pediatrics, Ayatollah Mousavi Hospital, Zanjan University of Medical Sciences, Zanjan, Iran.

\begin{tabular}{|c|c|}
\hline $\begin{array}{l}\text { Use your device to scan } \\
\text { and read the article online }\end{array}$ & Citation Arzanlou, M, Armanikian A, Saed O, Dobakhti F, Motamed N, Ahmadiafshar A. [Accuracy of Niacin Skin Flush Test \\
\hline 口ifiniti & $\begin{array}{l}\text { for Diagnosing Schizophrenia (Persian)] .Iranian Journal of Psychiatry and Clinical Psychology. 2021; 27(1):78-91. http://dx.doi. } \\
\text { org/10.32598/ijpcp.27.1.3144.1 }\end{array}$ \\
\hline 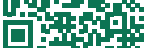 & doi http://dx.doi.org/10.32598/ijpcp.27.1.3144.1 \\
\hline
\end{tabular}

\section{(i) (3)}

Received: 03 Jul 2019

Accepted: 19 Aug 2020

Available Online: 01 Apr 2021

Key words:

Schizophrenia, Niacin Sensitivity, Specificity, Predictive Value

\section{A B S T R A C T}

Objectives This study aims to investigate niacin sensitivity in schizophrenia patients compared to healthy people and examine the accuracy of niacin skin flush test in diagnosing schizophrenia.

Methods This diagnostic study was conducted in 2018 in Zanjan, Iran. Three niacin concentrations $(0.001$ $\mathrm{M}, 0.01 \mathrm{M}$, and $0.1 \mathrm{M}$ ) was first applied topically to the skin of 36 schizophrenia patients and 33 healthy controls. Flush responses were evaluated at 10 and $15 \mathrm{~min}$ after application. Sensitivity, specificity, Positive Predictive Value (PPV), and Negative Predictive Value (NPV) of the test were measured for each niacin concentration and evaluation time.

Results At $10 \mathrm{~min}$, the highest test accuracy was reported when $0.001 \mathrm{M}$ niacin solution was used (Sensitivity $=94 \%$, specificity $=50 \%, P P V=51 \%$, and NPV= 94\%). At $15 \mathrm{~min}$, the highest test accuracy was observed at 0.01 concentration (Sensitivity $=52 \%$, specificity $=92 \%, P P V=79 \%$, and NPV $=77 \%$ )

Conclusion Flush responses to niacin is more impaired in patients with schizophrenia. Therefore, niacin can be considered as a biological marker of schizophrenia and can be used for its diagnosis.

\section{Extended Abstract}

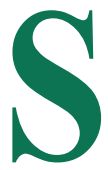

\section{Introduction}

chizophrenia is a severe mental disorder with high economic burden leading to emotional and social withdrawal with a symptoms such auditory hallucinations, catatonia, and delusions $[9$, 33]. Early identification of individuals with schizophrenia is important to treat them [12]. A high percentage of patients with schizophrenia have an attenuated or absent flush reaction compared to normal people $[32,34]$. Niacin skin flush test has been proposed as a possible simple biochemical test for identification of schizophrenia patients. There is no agreement on the predictive power of niacin skin flush test for identification of schizophrenia patients. Several studies have shown a diminished flush response to applied niacin in most schizophrenia patients, while some studies failed to show any difference [30]. This study aims to investigate niacin sensitivity in schizophrenia patients compared to healthy controls in order to examine the accuracy of niacin skin test results in these patients.

* Corresponding Author:

Mohammad Arzanlou

Address: Department of Psychiatry, School of Medicine, Zanjan University of Medical Sciences, Zanjan, Iran.

Tel: +98 (914) 4619095

E-mail: darmangar52@gmail.com 


\section{Methods}

This diagnostic study was conducted in 2018 on 36 schizophrenia patients aged 20-50 years and 33 healthy controls. Schizophrenia patients who were not in first episode of the disease were recruited from those refereed to psychiatric clinics or hospitals in Zanjan, Iran. Healthy controls were recruited from non-faculty hospital staff through an interview who had no history of major psychiatric disorders. The exclusion criteria were: Comorbid mental disease, acute medical condition, fever, metabolic, rheumatic, allergic, and thyroid diseases, use of mood stabilizer or blood pressure medications, use of haloperidol drugs in the past one year, use of nonsteroidal anti-inflammatory drugs or corticosteroids in the past two weeks, and use of antihistamine or drugs with antihistaminic effect in the past 10 days. Informed written consent was obtained from all participants before collecting data.

We applied niacin patches of three concentrations $(0.001$ $\mathrm{M}, 0.01 \mathrm{M}$, and $0.1 \mathrm{M}$ ) of aqueous methyl nicotinate topically to the forearm skin of all subjects for $5 \mathrm{~min}$. Flush responses were rated 10 and $15 \mathrm{~min}$ after application. The strength of flush responses was rated as $0=$ No response and $1=$ With response according to the literature [32]. Sensitivity, specificity, Positive Predictive Value (PPV), and Nega- tive Predictive Value (NPV) of the test were reported for each concentration and evaluation times. Niacin skin flush test was conducted by an expert who was unaware of the study groups. The categorical variables were compared between groups by chi-square test. Statistical analyses were performed in SPSS V. 24 software.

\section{Results}

The Mean \pm SD age of schizophrenic patients was $36.72 \pm 8.72$ years ( $55.6 \%$ male), and for the healthy control group it was $35.33 \pm 7.31$ years ( $54.5 \%$ male). There was no significant difference in terms of age and gender between the two groups at baseline, but their education level and employment status were significantly different (higher in controls). Frequency of response and no response in each group at different time points (10 and $15 \mathrm{~min}$ ) and concentrations $(0.1 \mathrm{M}, 0.01 \mathrm{M}$, and $0.001 \mathrm{M})$ are presented in Table 1 . Accuracy parameters of the niacin skin flush test results in differentiating schizophrenia patients from controls are presented in Table 2.

\section{Discussion and Conclusion}

Our findings were consistent with the results of other studies regarding that flush response to niacin patches was

Table 1. Frequency of flush response/non-response to niacin at different concentrations and time points

\begin{tabular}{|c|c|c|c|c|c|}
\hline \multirow{3}{*}{ Group } & \multirow{3}{*}{ Solution } & \multirow{3}{*}{ Reaction } & \multicolumn{2}{|c|}{ No. (\%) } & \multirow{3}{*}{$\mathrm{P}^{*}$} \\
\hline & & & \multicolumn{2}{|c|}{ Time } & \\
\hline & & & $10 \mathrm{~min}$ & $15 \mathrm{~min}$ & \\
\hline \multirow{9}{*}{ Schizophrenia } & \multirow{3}{*}{$0.1 \mathrm{M}$} & No response & $9(25)$ & $6(16.7)$ & \multirow{3}{*}{$<0.001$} \\
\hline & & & & & \\
\hline & & Response & $27(75)$ & $30(83.3)$ & \\
\hline & \multirow{3}{*}{$0.01 \mathrm{M}$} & No response & $21(58.3)$ & 19 (52.7) & \multirow{3}{*}{$<0.001$} \\
\hline & & & & & \\
\hline & & Response & $15(41.7)$ & $17(47.3)$ & \\
\hline & \multirow{3}{*}{$0.001 \mathrm{M}$} & No response & 34 (94.4) & $32(88.8)$ & \multirow{3}{*}{0.036} \\
\hline & & & & & \\
\hline & & Response & $2(5.6)$ & $4(11.2)$ & \\
\hline \multirow{9}{*}{ Healthy control } & \multirow{3}{*}{$0.1 \mathrm{M}$} & No response & $4(12.1)$ & $2(6.06)$ & \multirow{3}{*}{$<0.001$} \\
\hline & & & & & \\
\hline & & Response & 29 (87.9) & 31 (93.94) & \\
\hline & \multirow{3}{*}{$0.01 \mathrm{M}$} & No response & $9(27.3)$ & $1(3.03)$ & \multirow{3}{*}{$<0.001$} \\
\hline & & & & & \\
\hline & & Response & 24 (72.7) & 32 (96.97) & \\
\hline & \multirow{3}{*}{$0.001 \mathrm{M}$} & No response & $18(54.5)$ & $14(42.4)$ & \multirow{3}{*}{$<0.001$} \\
\hline & & & & & \\
\hline & & Response & $15(45.5)$ & $19(57.6)$ & \\
\hline
\end{tabular}

*Friedman test. 
Table 2. Accuracy of the niacin skin flush test in differentiating schizophrenia patients from controls

\begin{tabular}{|c|c|c|c|c|c|}
\hline \multirow{2}{*}{ Time } & \multirow{2}{*}{ Solution } & \multicolumn{4}{|c|}{$\%$} \\
\hline & & Sensitivity & Specificity & PPV & NPV \\
\hline \multirow{3}{*}{$10 \mathrm{~min}$} & $0.1 \mathrm{M}$ & 25 & 89 & 56 & 68 \\
\hline & $0.01 \mathrm{M}$ & 58 & 67 & 50 & 74 \\
\hline & $0.001 \mathrm{M}$ & 94 & 50 & 51 & 94 \\
\hline \multirow{3}{*}{$15 \mathrm{~min}$} & $0.1 \mathrm{M}$ & 16 & 95 & 66 & 67 \\
\hline & $0.01 \mathrm{M}$ & 52 & 92 & 79 & 77 \\
\hline & $0.001 \mathrm{M}$ & 88 & 67 & 60 & 91 \\
\hline
\end{tabular}

Iranian Journal of
PSYCHIATRY AND CUNICAL PSYCHOLOGY

significantly impaired in patients with schizophrenia compared to normal healthy population. We found that the use of $0.001 \mathrm{M}, 0.01 \mathrm{M}$, and $0.1 \mathrm{M}$ solutions resulted in different test accuracy results, where better sensitivity and NPV were observed at $0.001 \mathrm{M}$ solution. The increased activity of phospholipase A2 in schizophrenic patients which downregulates the level of arachidonic acid and breakdown membrane phospholipids, may be responsible for reduced responses to niacin $[32,34,35]$, but this mechanism does not seem to be a reason in all schizophrenic patients. Previous studies have shown that that only a small group of schizophrenic patients shows impaired flush response to niacin $[36,37]$. Therefore, the skin flush response may be an endo-phenotype, rather than a specific marker for schizophrenia. We concluded that the niacin skin flush test is a valuable diagnostic tool for schizophrenia. Better understanding of this biomarker can result in early identification of schizophrenia for conducting interventions.

\section{Ethical Considerations}

\section{Compliance with ethical guidelines}

This study was approved by the Ethical Committee of the Zanjan University of Medical Sciences (IR.ZUMS. REC.1397.125). Informed written consent was obtained from all participants before collecting data. All ethical principles are considered in this article. The participants were informed about the purpose of the research and its implementation stages. They were also assured about the confidentiality of their information and were free to leave the study whenever they wished, and if desired, the research results would be available to them.
Funding

This research did not receive any grant from funding agencies in the public, commercial, or non-profit sectors.

\section{Authors contributions}

Conceptualization, methodology, validation, analysis data: All authors; Investigation, preparation, writing-review \& editing: Mohammad Arzanlou; Project administration: Alireza Armanikian.

\section{Conflicts of interest}

The authors declared no conflicts of interest.

\section{Acknowledgements}

The authors would like to thank the psychologists and psychiatrists who helped identify schizophrenia patients, the personnel of Shahid Beheshti Hospital in Zanjan for cooperation, and all participants of the study. 


\section{تعيين ارزش ييش بينى كنئدى تست حساسيت يوستى ثياسين براى تشخيص بيمارى اسكيزوفرنيا}

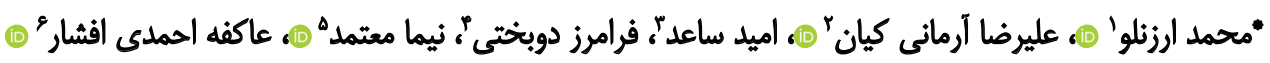

1. كروه روانيزشكى، دانشكده يزشكى، دانشكاه علوميزشكى زنجان، زنجان، ايران.

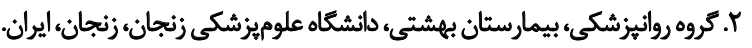

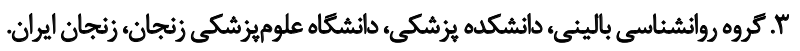

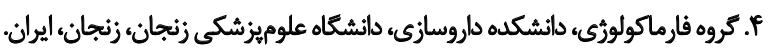

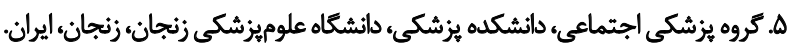

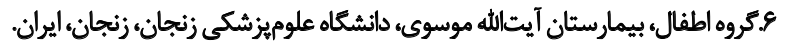

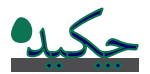

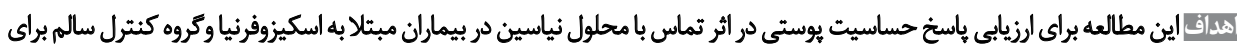

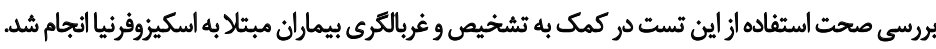

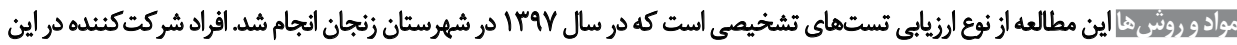

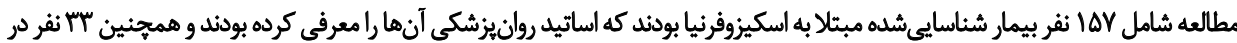

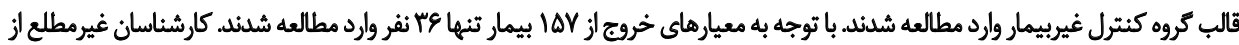

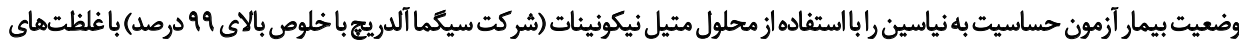

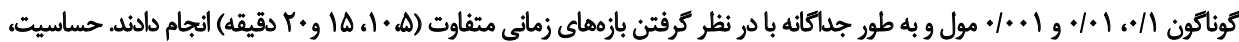

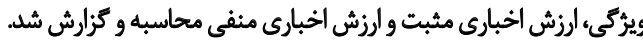

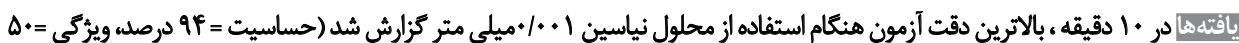

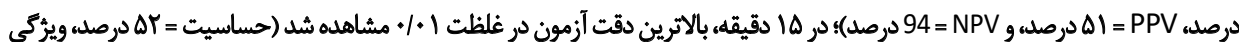

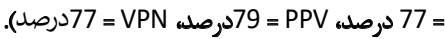

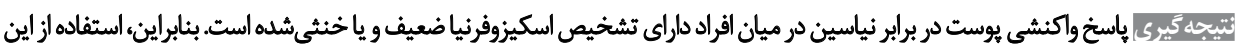

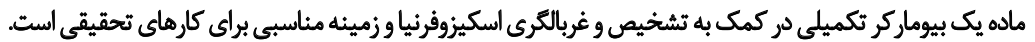

تاريخ دريافت: Tاتير

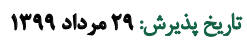

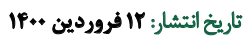

كليدواثهها:

اسكيزوفرنياء نياسين،

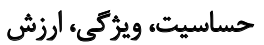

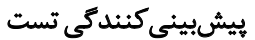

خود اختصاص داده است [ه-1 ]. طبق يُنجمين نسخه راهنماى

مقلمه

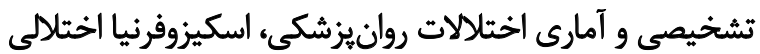

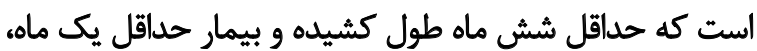

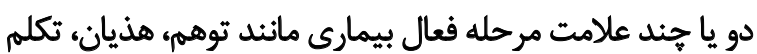

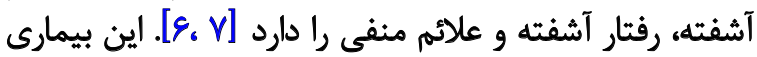

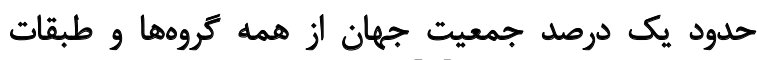

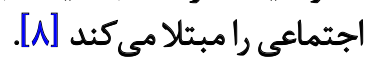

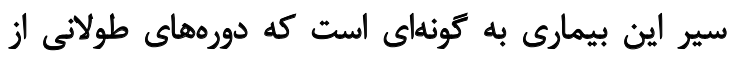

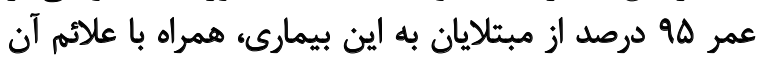

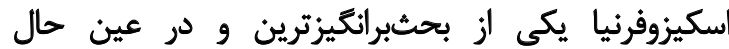

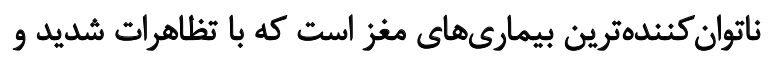

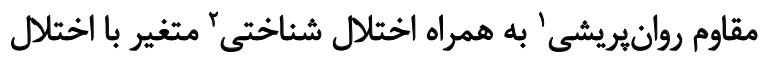

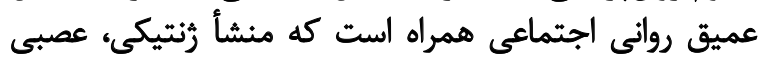

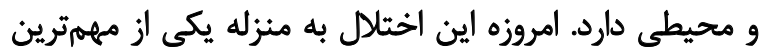

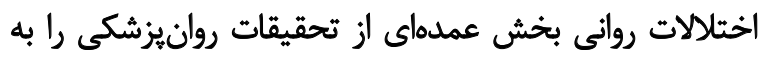

1. Psychosis

2. Cognitive Disorder

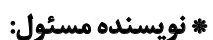

محمد ارزنلو

نشانىي: زنجان، دانشعاه علوميزٔشكى زنجان، دانشكده يزشكى، كروه روانيزشكى.

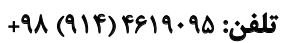
يست الكترونيكي: darmangar52@gmail.com 
باشد. اين متابوليسم نقش كليدى در ياسخ واكنشى يوست به به به

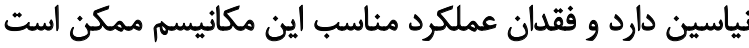

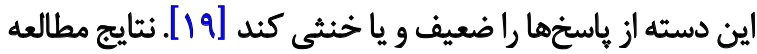

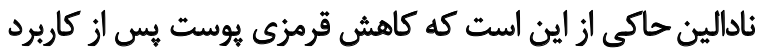

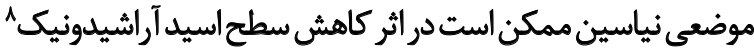

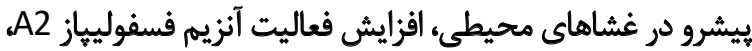

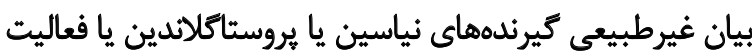

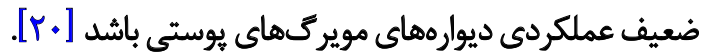

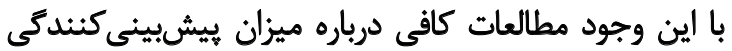

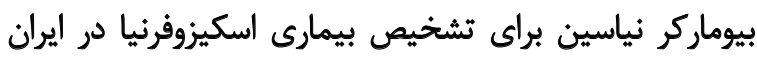

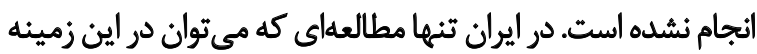

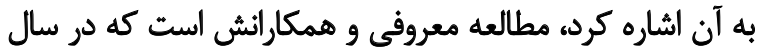

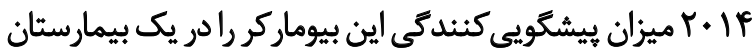

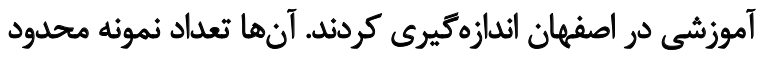

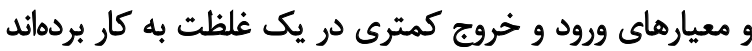

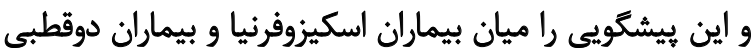

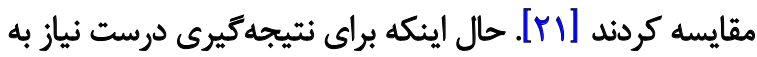

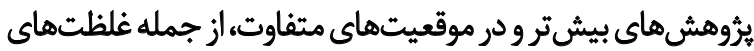

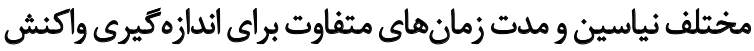

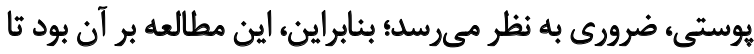

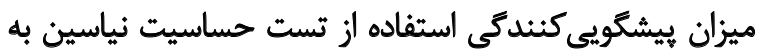

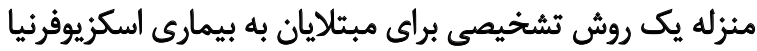

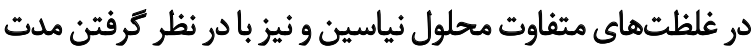

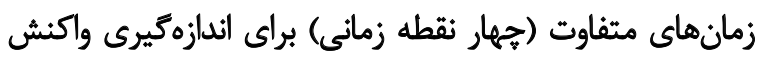

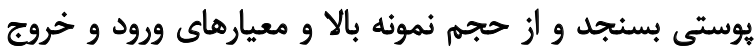

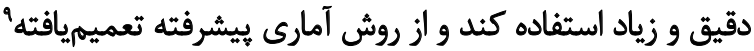

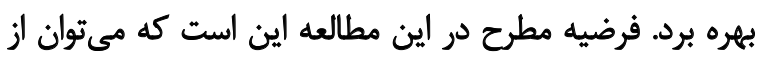

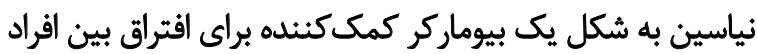

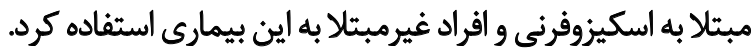

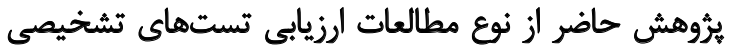

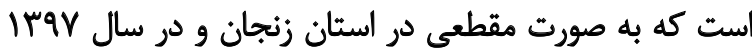

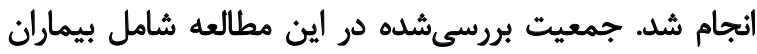

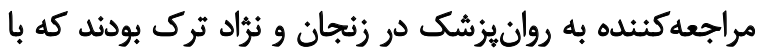

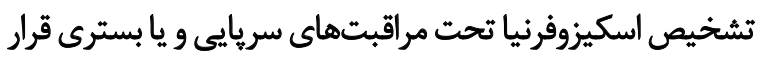

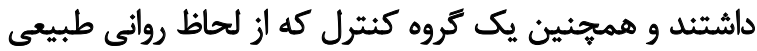

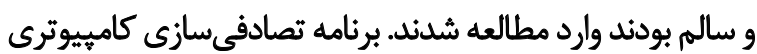

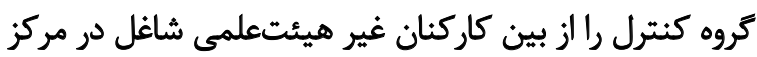

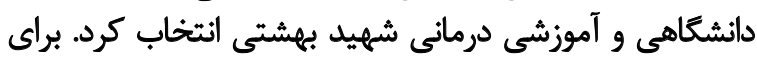

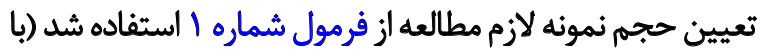

8. Arachidonic acid

9. Generalized estimation equation (GEE)
سيرى مى شود و حدود •ه درصد تختهائ بيمارستانى مراكز

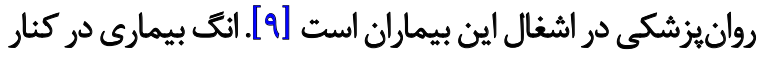

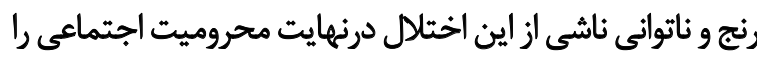

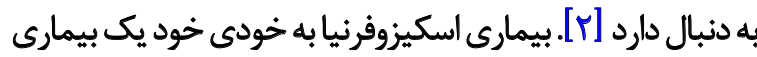

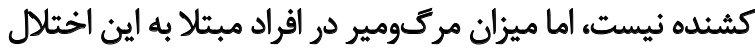

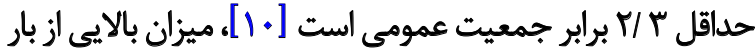

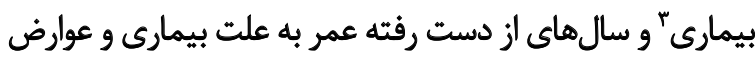

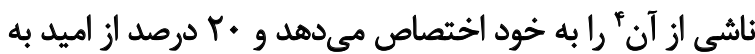

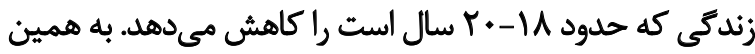

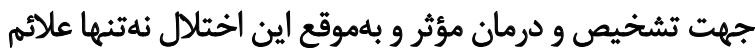

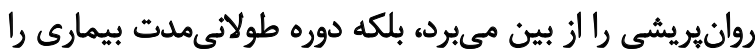

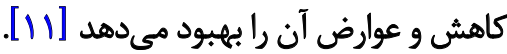
امروزه تنها راه تشخيص بيمارى بر اساس علائم ذكرشده در بر بردي

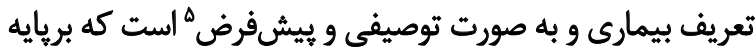

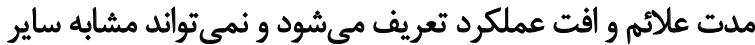

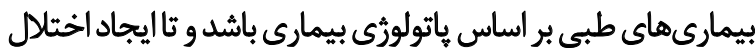

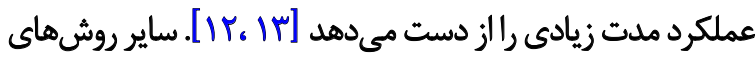

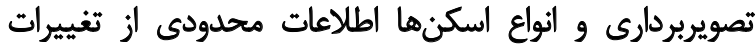

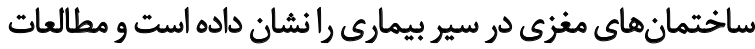

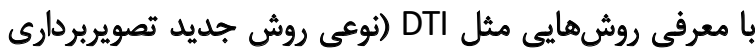

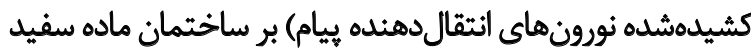

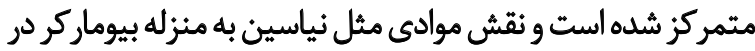

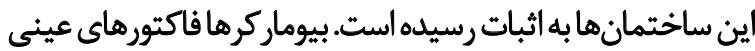

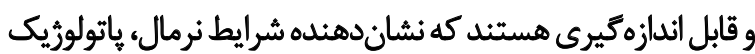

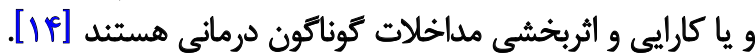

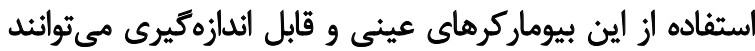

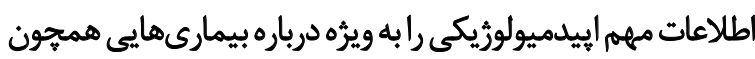

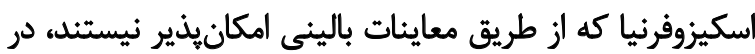

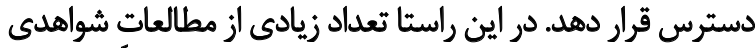

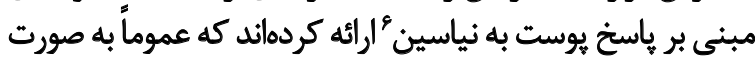

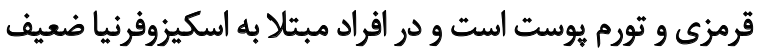

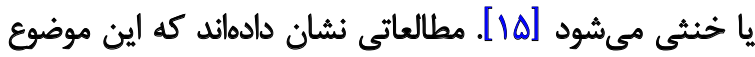

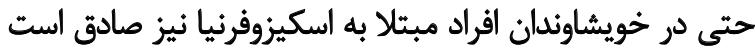

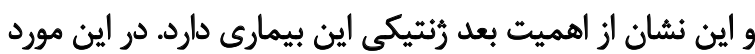

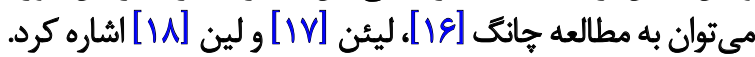

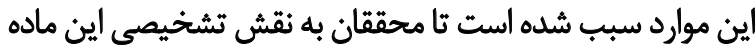

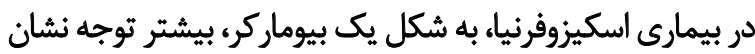

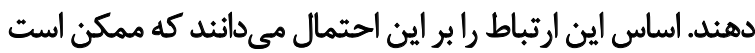

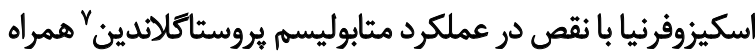

3. Global Burden of Disease

4. Disability Adjusted Life Years

5. Menu-Based

6. Niacin (nicotinic acid)

7. Prostaglandin metabolism 


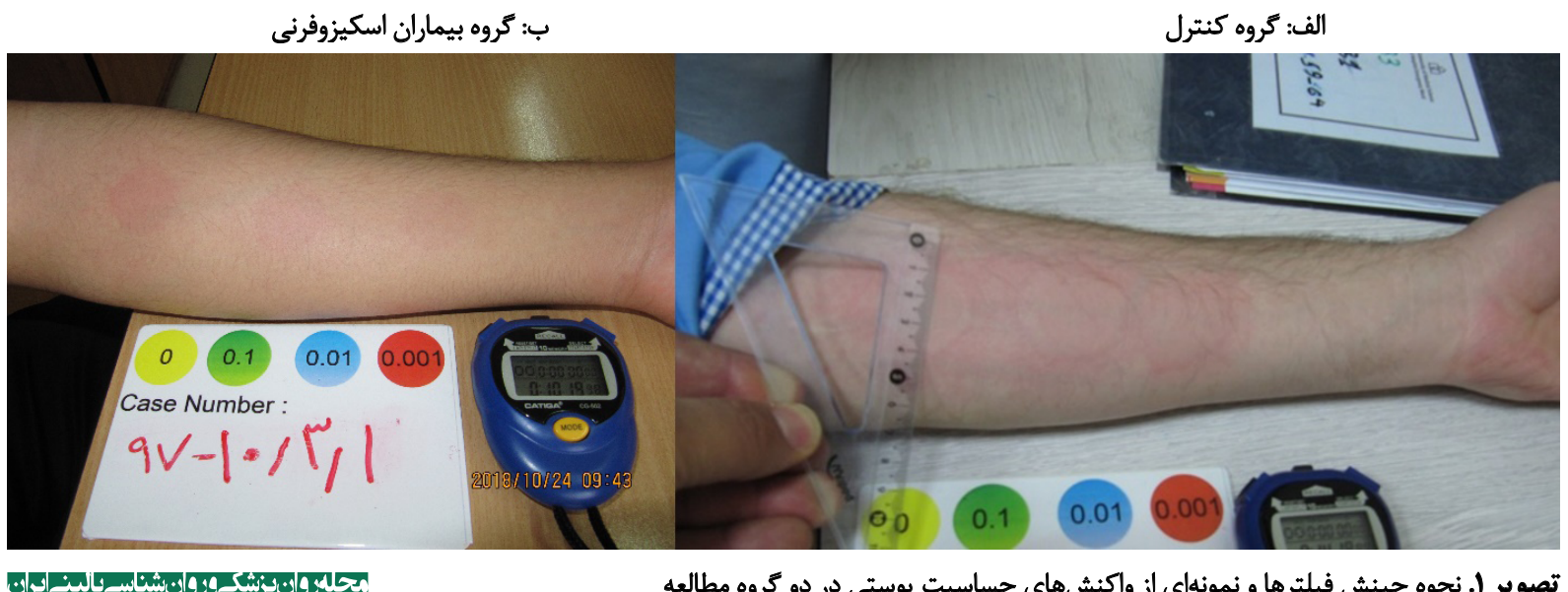

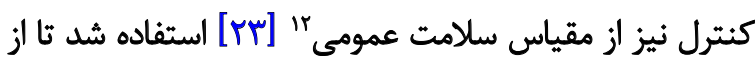

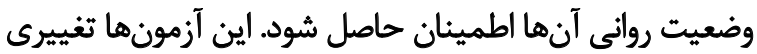

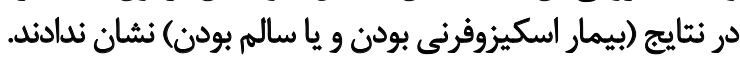

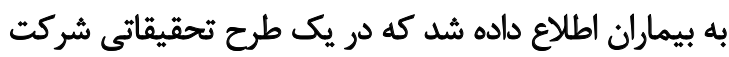

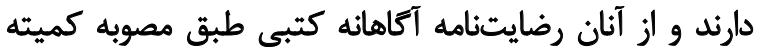

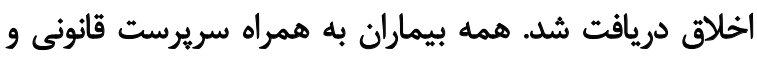
با اطلاع و معرفى استادان معالج وارد مطالعه شداند و ورئ مجري

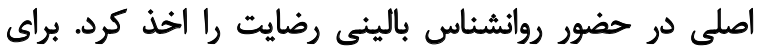

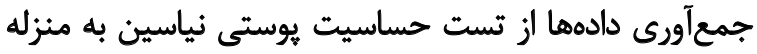

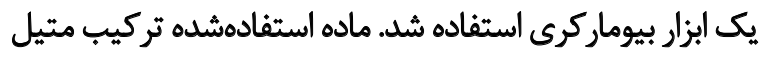

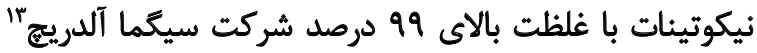

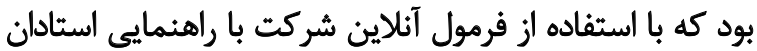

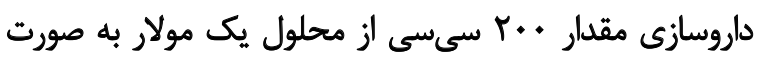

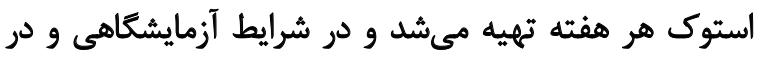

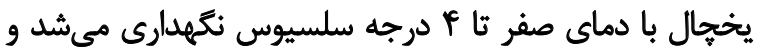

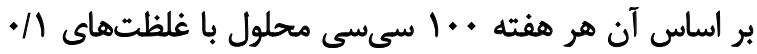

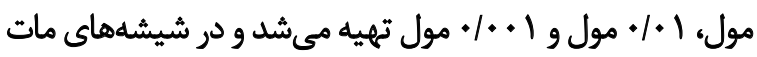

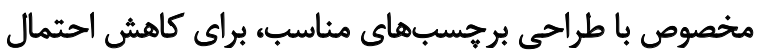

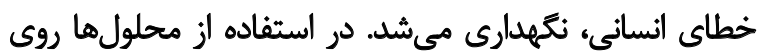

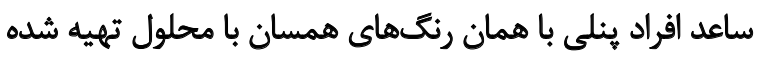

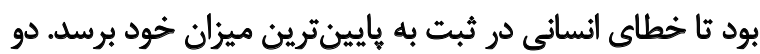

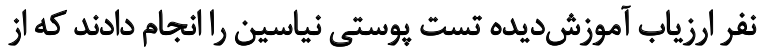

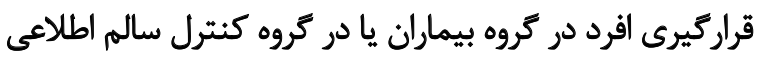

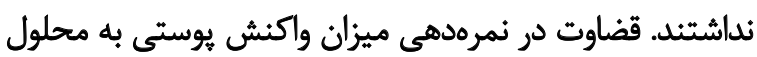

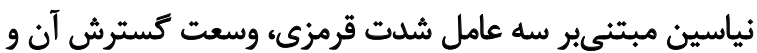

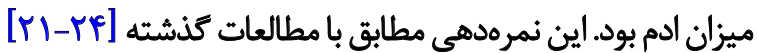

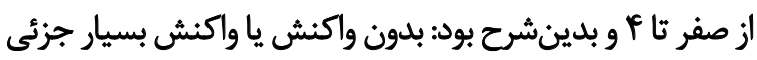

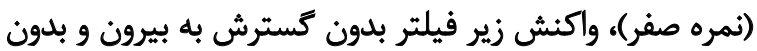

12. The General Health Questionnaire-28 items (GHQ) 13. Sigma-Aldrich
سطح خطاي نوع يك هـ/ و قدرت مطالعه 19 /•).

$$
\frac{\left(Z_{1_{-a} / 2}+Z_{1_{-\beta}}\right)^{2}\left(S D_{1}^{2}+S D_{2}^{2}\right)}{\left(\mu_{1}-\mu_{2}\right)^{2}}
$$

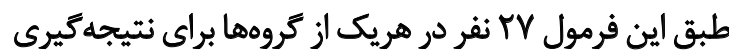

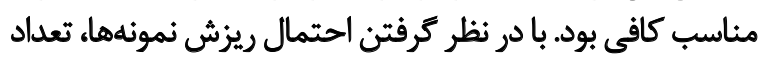

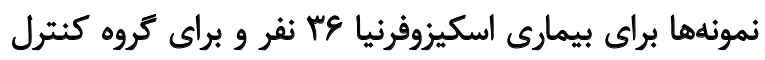

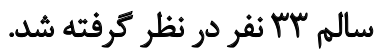

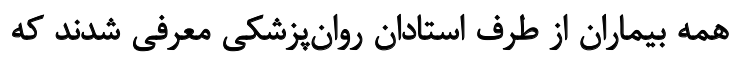

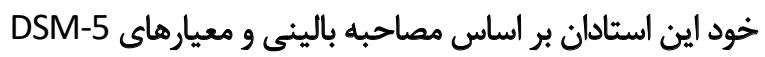

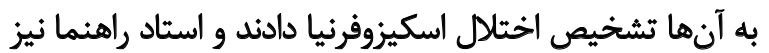

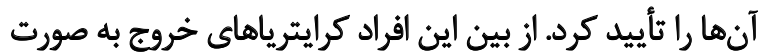

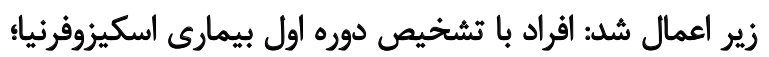

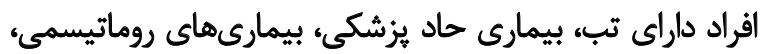

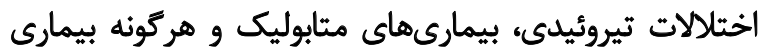

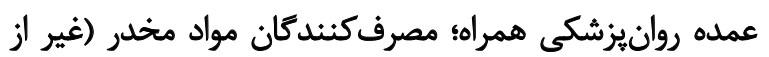

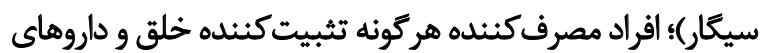

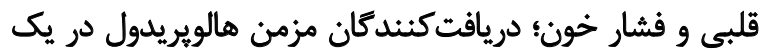

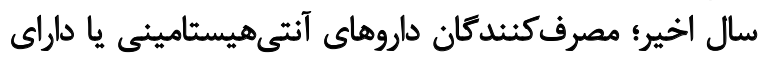

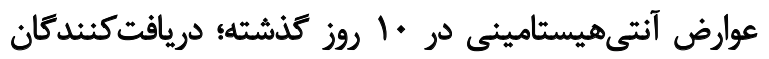

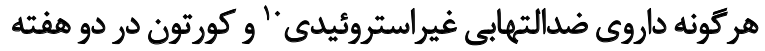

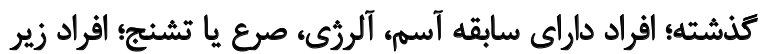

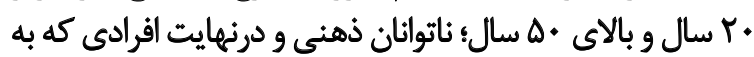

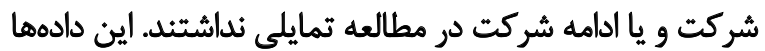

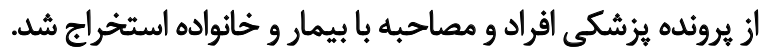

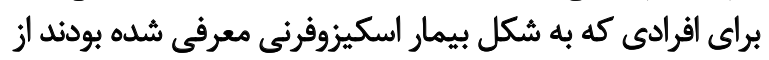

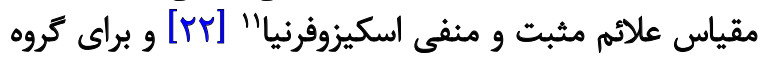




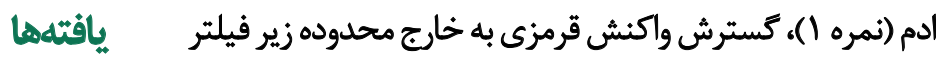

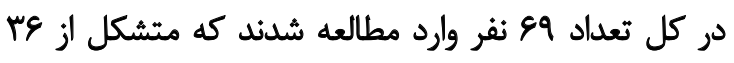

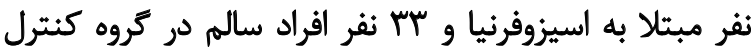

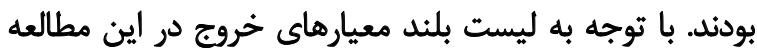

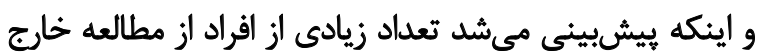

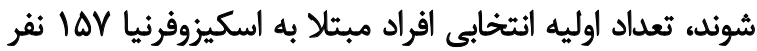

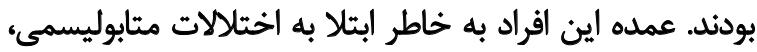

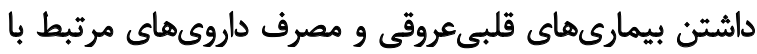

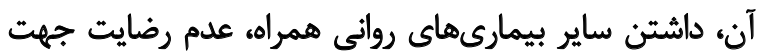

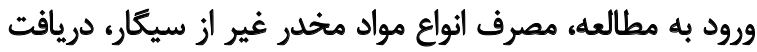

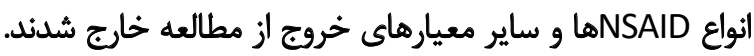

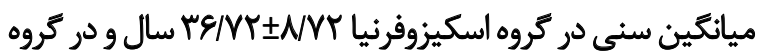
كنترل I S

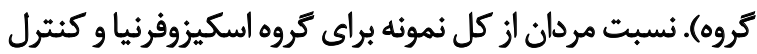

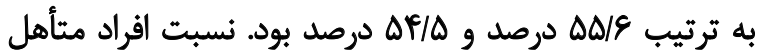

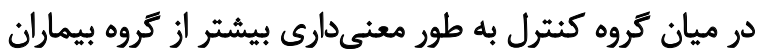

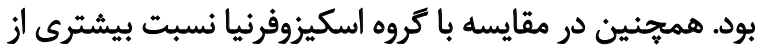

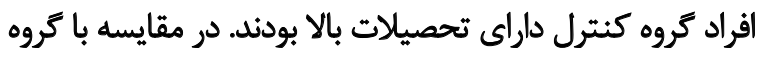

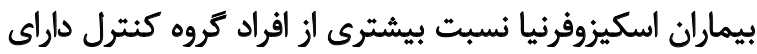

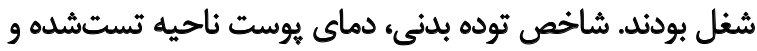

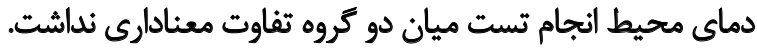

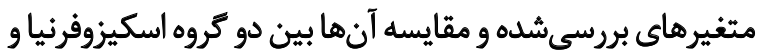
كنترل در جدول شماره r نشان داده شده است.

با توجه به اينكه از مقياس علائم مثبث و منفى اسكيزوفرنيا

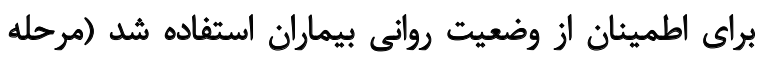

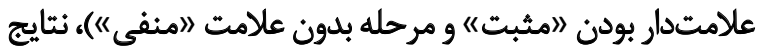

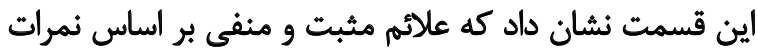

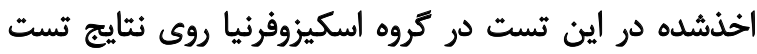

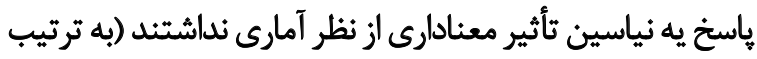

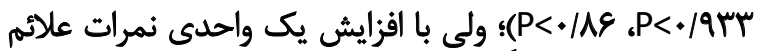

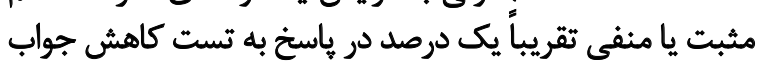

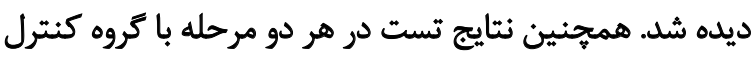

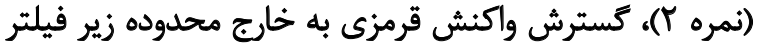

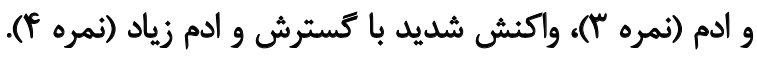

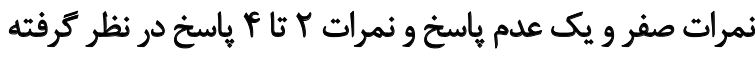

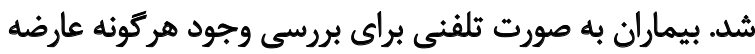

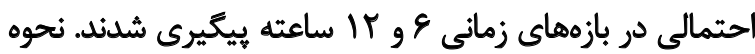

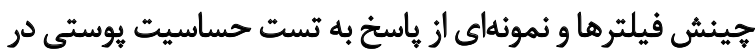
هر دو كروه در تصوير شماره إنشان داده شده است.

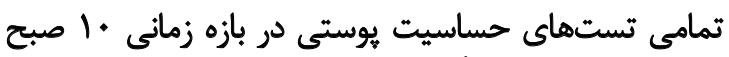

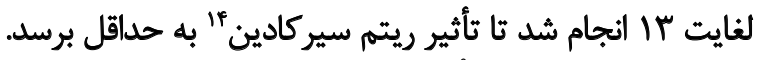

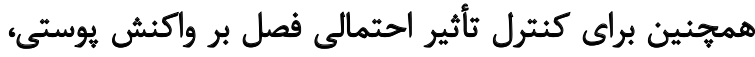

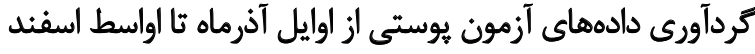

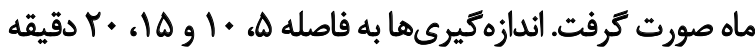
يس از تماس يوستى انجام و ثبت شد أندان

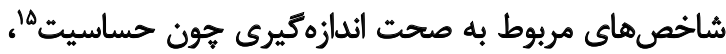

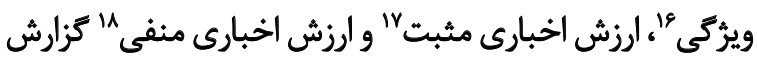

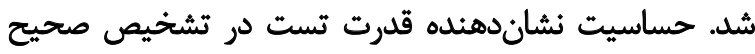

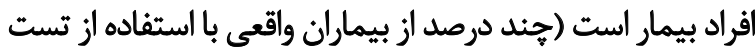

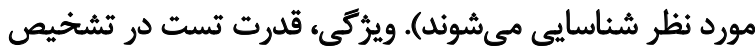

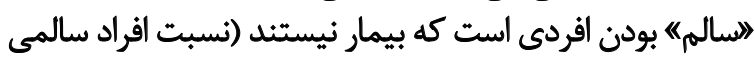

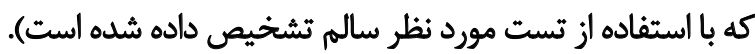

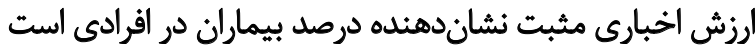

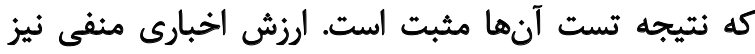

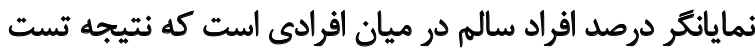

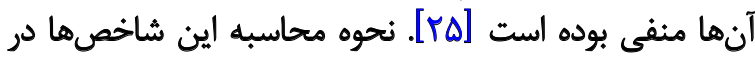

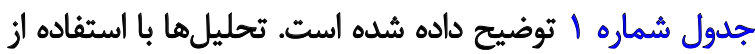

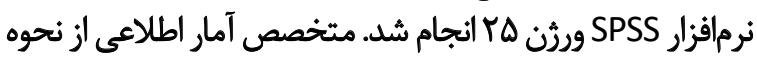

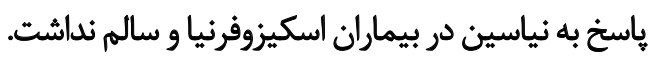

14. Circadian rhythm

15. Sensitivity

16. Specificity

17. Positive predictive value

18. Negative predictive value

جدول ا. محاسبه حساسيت، ويدُّى، ارزش اخبارى مثبت و ارزش اخبارى منفى

\begin{tabular}{|c|c|c|}
\hline نتيجه تست & فرد بيمار است & فرد بيمار نيست \\
\hline نتيجه تست مثبت است & B & A \\
\hline \multirow[t]{2}{*}{ نتيجه تست منفى است } & c & D \\
\hline & 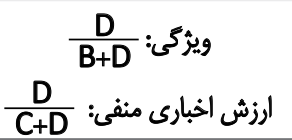 & 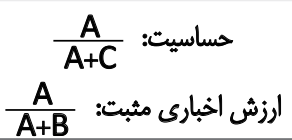 \\
\hline
\end{tabular}


جدول r. توزيع فراوائى متغيرهاى جمعيتشناختى در كروهاى مطالعه

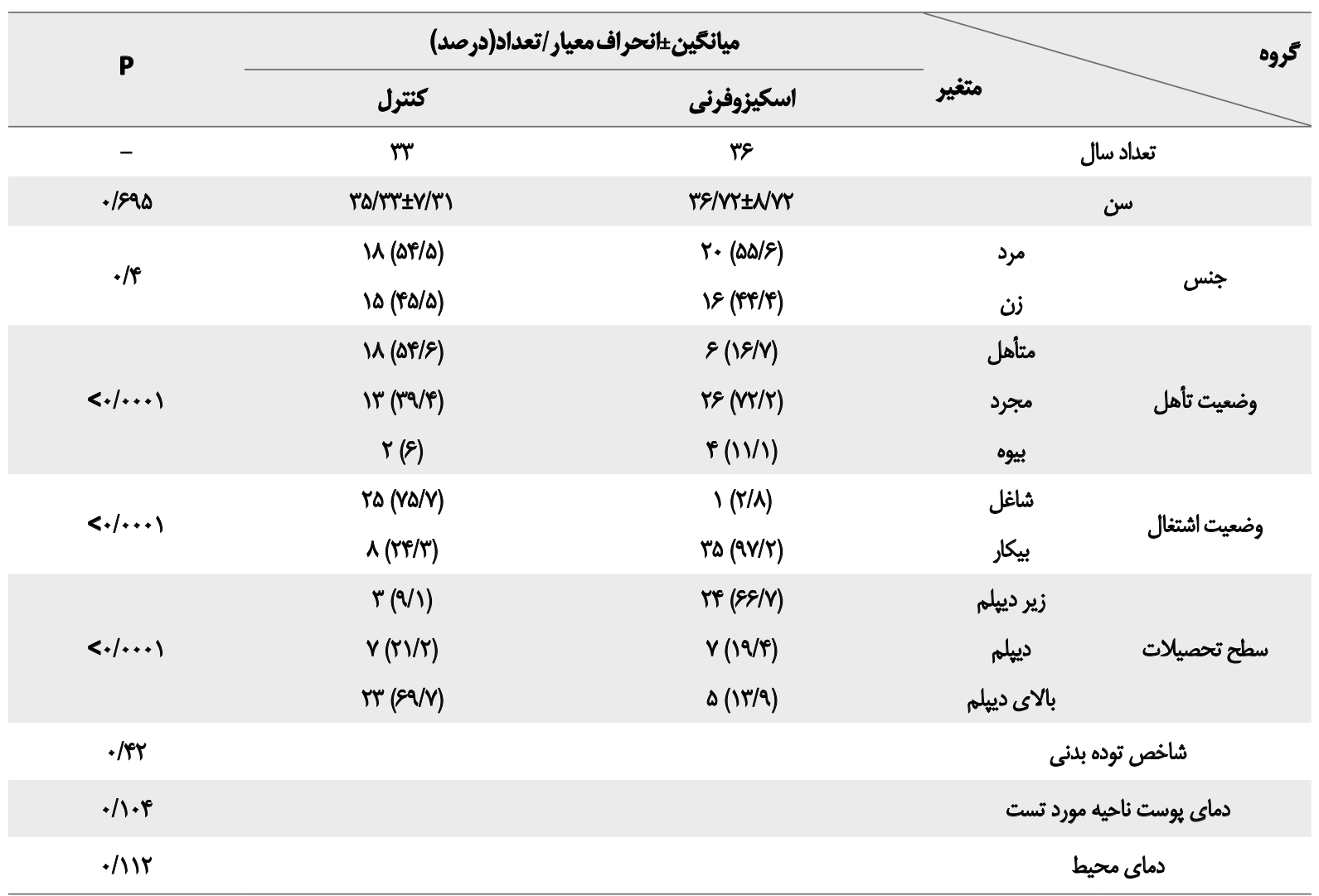

جدول ب. توزيع فراوائي ياسخ و نبود ياسخ به تست يوستى نياسين درغلظتها و زمانهاى مطالعه به تفكيك كروهها

\begin{tabular}{|c|c|c|c|c|c|}
\hline \multirow{3}{*}{$\mathbf{P}$} & \multicolumn{2}{|c|}{ زمان الرزيابي (دقيقه) } & \multirow{3}{*}{ درجه قرمزى بوست } & \multirow{3}{*}{ غلظت } & \multirow{3}{*}{ كرو } \\
\hline & 10 & 1. & & & \\
\hline & \multicolumn{2}{|c|}{ تعداد (درصد) } & & & \\
\hline$<\cdot 1 \cdot .+1$ & $\begin{array}{c}\&(\mid \& / V) \\
r+(A r / r)\end{array}$ & $\begin{array}{l}q(T \Delta) \\
r V(V \Delta)\end{array}$ & نيود هاستخ & .11 & \multirow{3}{*}{ اسكيزوفرنى } \\
\hline$<+1+\infty 1$ & $\begin{array}{l}19(\Delta r / V) \\
1 Y(F V / Y)\end{array}$ & $\begin{array}{l}r(\Delta N H) \\
\|(F M N)\end{array}$ & نبود باستخ & $.1+1$ & \\
\hline e & $\begin{array}{l}H(M N) \\
F(I / / Y)\end{array}$ & $\begin{array}{l}m(q f / f) \\
r(\Delta / \varepsilon)\end{array}$ & نبود باستخ & $.1+.1$ & \\
\hline$<\cdot 1 \cdot .1$ & $\begin{array}{c}r(q / .9) \\
r(q m / q f)\end{array}$ & $\begin{array}{l}P(\mathrm{Ir} / \mathrm{l}) \\
r q(\mathrm{Ar} / \mathrm{q})\end{array}$ & 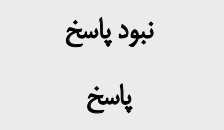 &.$/ 1$ & \multirow{3}{*}{ كتترل } \\
\hline$<\cdot 1 \cdot+1$ & $\begin{array}{c}I(r / \circ) \\
T(q / q V)\end{array}$ & $\begin{array}{l}Q(T V / T) \\
r F(N / Y)\end{array}$ & نيود باسنخ & $\cdot / \cdot 1$ & \\
\hline$<\bullet / \bullet+1$ & $\begin{array}{l}\text { If (FY/F) } \\
19(\Delta V / Y)\end{array}$ & $\begin{array}{l}M(\Delta f / \Delta) \\
1 \Delta(f \Delta / \Delta)\end{array}$ & نبود ياستخ & $.10+1$ & \\
\hline
\end{tabular}




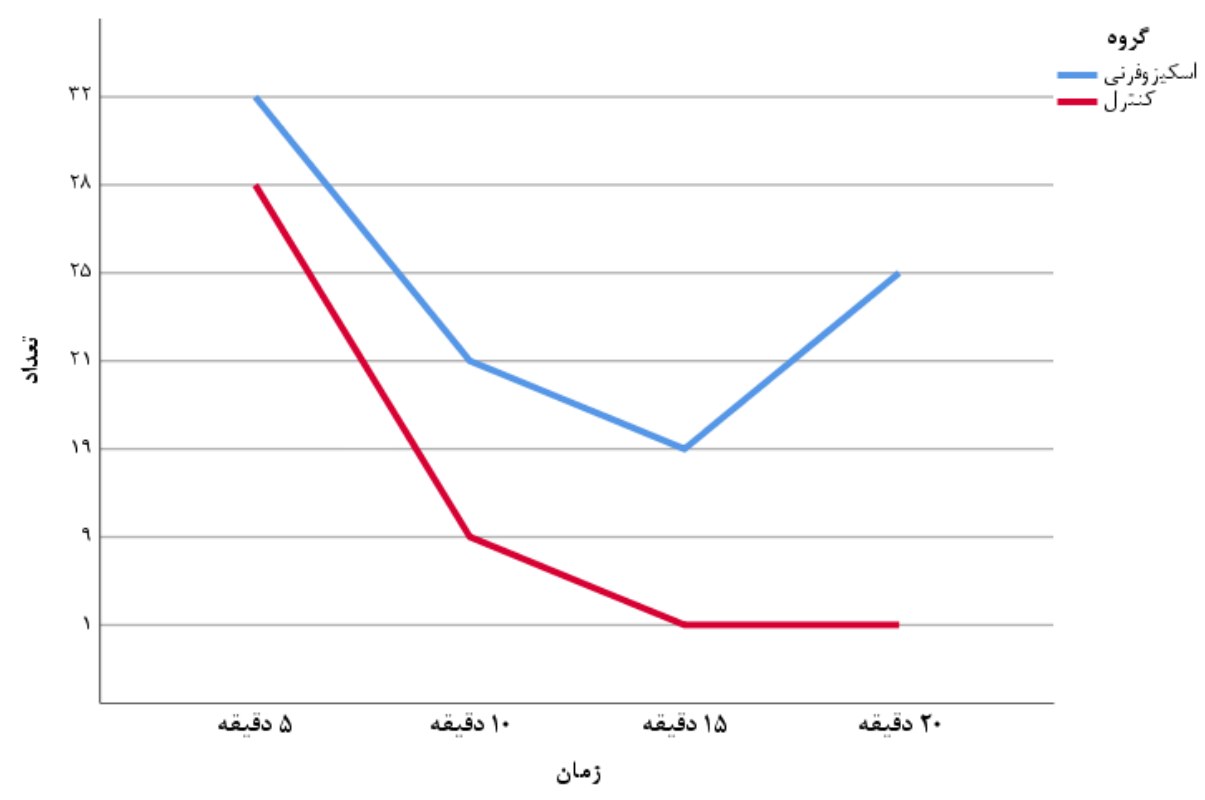

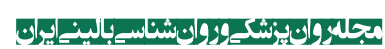

تصوير ז. منحنى نبود ياسخ در طى زمان در غلظت ا ٪/ مول

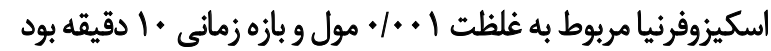

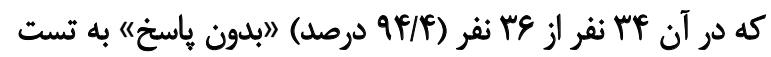

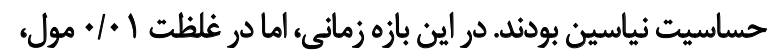

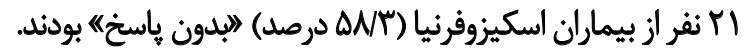

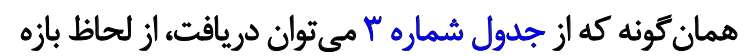

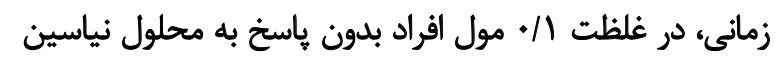

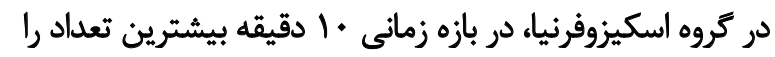

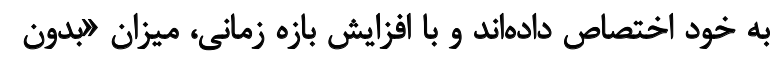

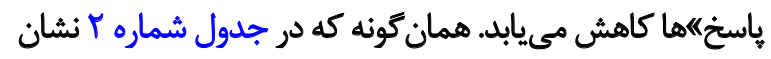

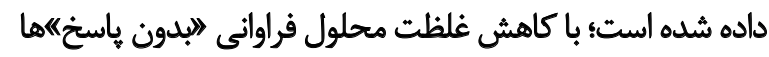

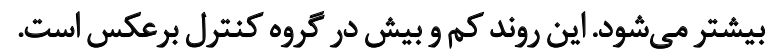

فراوانى ميزان ياسخ ندادن (يعنى امتياز صفر يا يك در رابطه

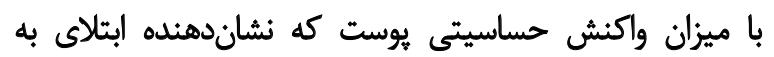

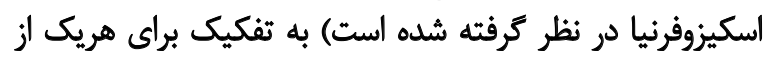

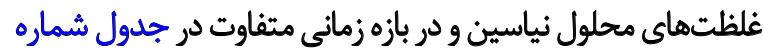

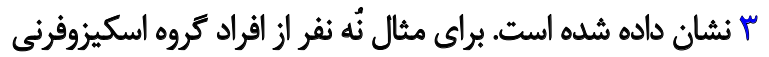

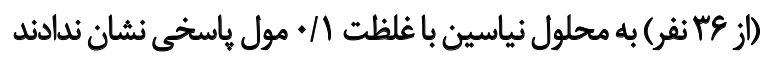

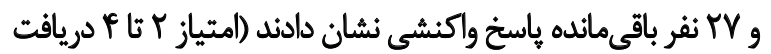

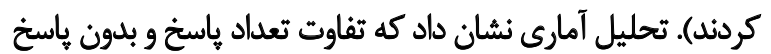

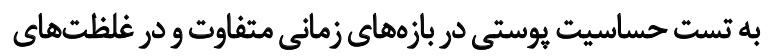

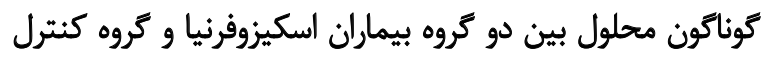

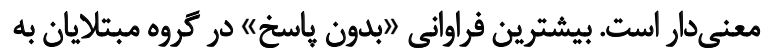

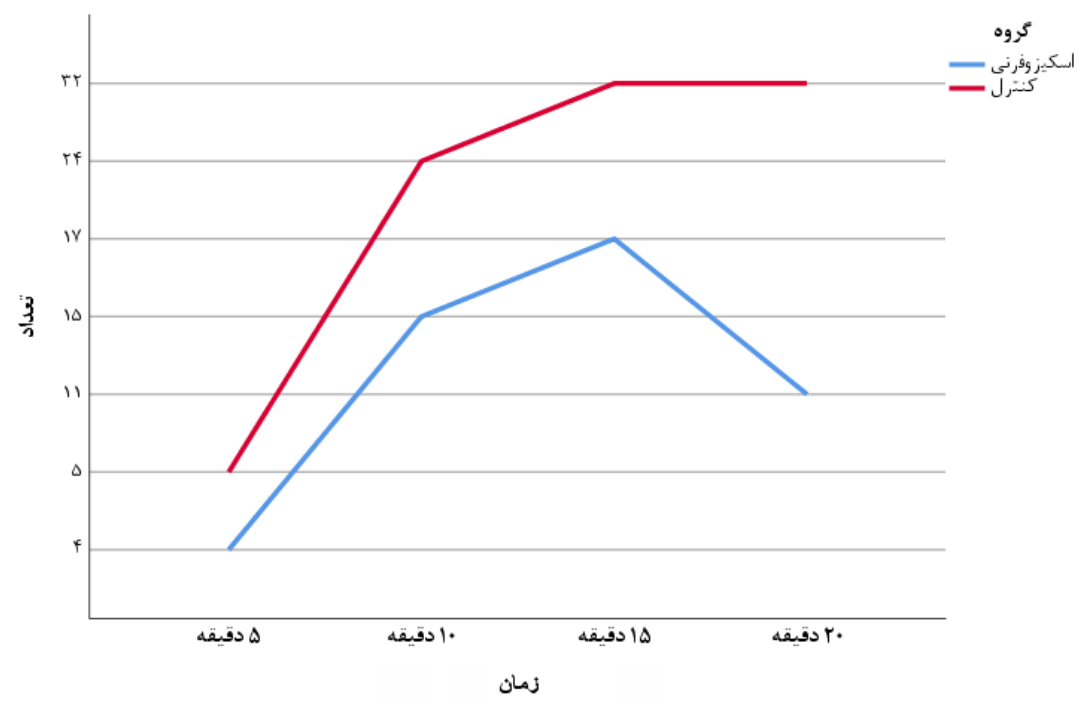




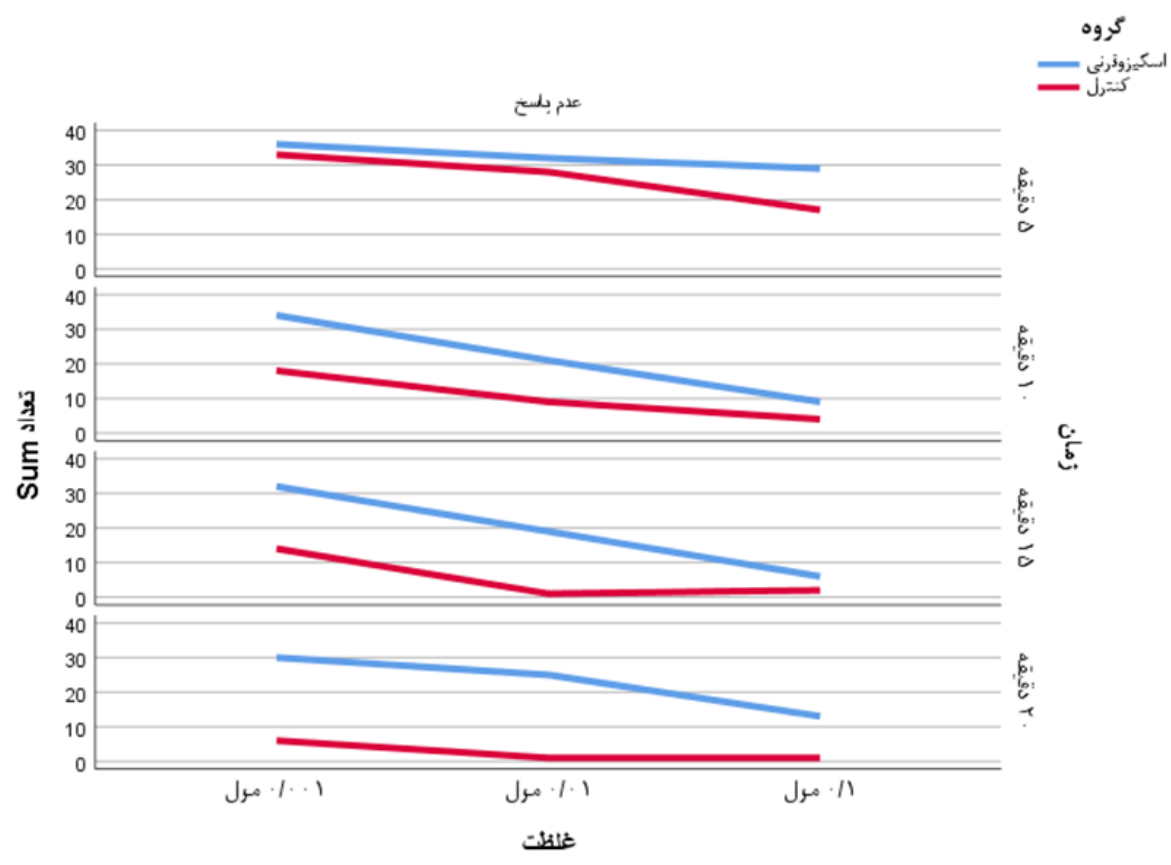

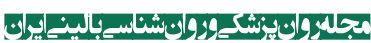

تصوير f. منحنى نبود ياسخ بر اساس غلظت

كروه اسكيزوفرنى كمتر (تصوير شماره ") است. بدون ياسخ در

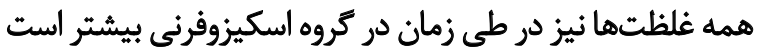

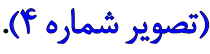

حساسيت، ويزّكى، ارزش اخبارى مثبت و ارزش اخبارى منفى

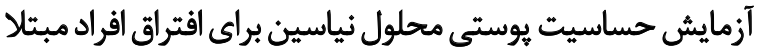

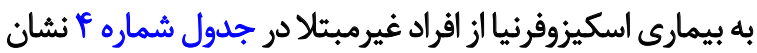
داده شده است. بيشترين مقادير حساسيت و ارزش ارئي اخباري

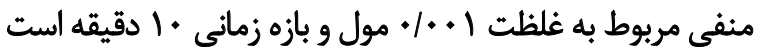

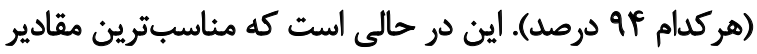

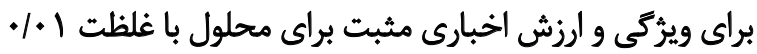

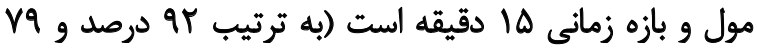

درصد).
نتايج مطالعه نشان داد كه ياسخ ندادن در غلظتهاي كونائون

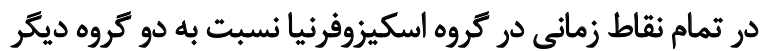
بيشتر است. متغير وابسته در جدول شماره ب يك متغير دو حالتى ياسخ

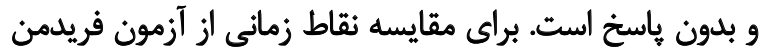

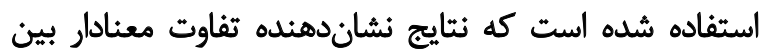

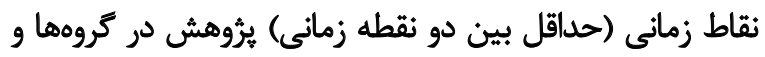

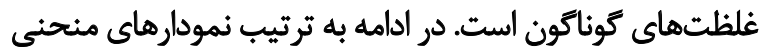

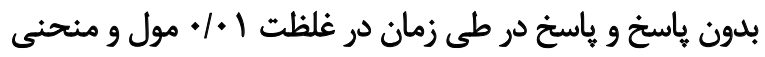

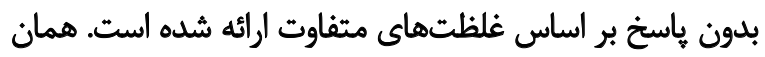

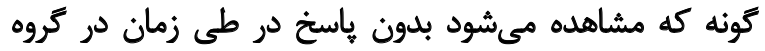

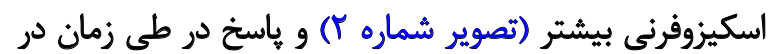
جدول f. ارزش بيش كويى كنئدى تست يوستى نياسين براي بيمارى اسكيزوفرنى

\begin{tabular}{|c|c|c|c|c|c|}
\hline الرزش اخبارى منفى & ارزش اخبارى مثبت & ويؤكى & حساسيت & غلظت محلول & زمان ارزيابى \\
\hline \multicolumn{6}{|c|}{ درصد (فاصله اطمينان) } \\
\hline$\cdot \operatorname{l} A \lambda(+/ \Delta A-+/ V A)$ & $\cdot|\Delta F(\cdot / \mu)-+| \lambda)$ & $\cdot / 199(\cdot / 11-+/ 99)$ & $\cdot / r \Delta(\cdot / 1+-\cdot / r q)$ & .11 & \\
\hline$\cdot M^{e}\left(\cdot / \mathscr{A l}^{\top}-\cdot / M \Delta\right)$ & $\cdot / \Delta \cdot\left(\cdot / /{ }^{e}-\cdot / 8 \Delta\right)$ & $.18 V(\cdot 1 \Delta s-. / N q)$ & $\cdot / \Delta A\left(\cdot / P T-\cdot / M^{e}\right)$ & .1 .1 & . أدقيقه \\
\hline.$/ A f(\cdot \mid \wedge \varepsilon-1)$ & $\cdot / \Delta)\left(\cdot / T^{q}-\cdot / \mathbb{R}^{w}\right)$ & $\cdot 10 \cdot(\cdot / \mathrm{T} \Lambda-\cdot / 8 T)$ &.$/ 9 P(\cdot / N-1)$ & .1 .01 & \\
\hline$\cdot 18 V(\cdot / \Delta V-\cdot N 8)$ &.$/ \notin 8(. / \% \Delta-+/ 9 Y)$ &.$/ 9 \Delta(\cdot / 9-1)$ & $\cdot / \Lambda \&\left(\cdot /+\varphi_{-} \cdot / T \Lambda\right)$ & .11 & \\
\hline$\cdot / W(\cdot / \& A-\cdot / A V)$ &.$/ N q(\cdot / 8 Y-\cdot / 9 \Delta)$ &.$/ 9 r(\cdot / 1 \Delta-\cdot / 24)$ & $\cdot / \Delta r(\cdot / r g-. / 89)$ & $.1+1$ & ها دقيقه \\
\hline.$/ 91(. / 14-. / 99)$ & $\cdot / 8 \cdot\left(\cdot / / 2 V-\cdot M^{2}\right)$ & $.18 v(.108-. / N 9)$ &.$/ M(\cdot / V A-. / 99)$ & $.1 . .1$ & \\
\hline
\end{tabular}


به محلول نياسين در بيماران اسكيزوفرنيا مطالعه لين و همكاران

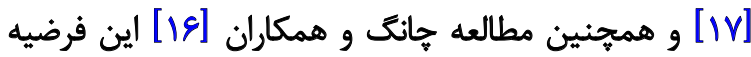

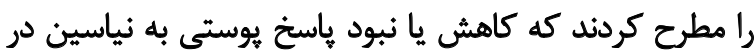

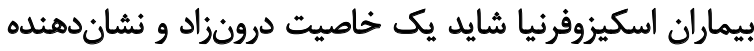

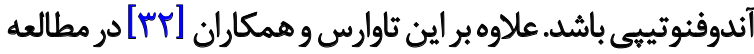

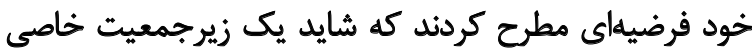

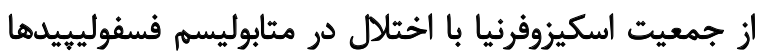

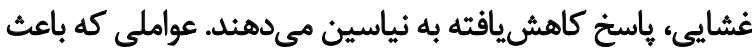

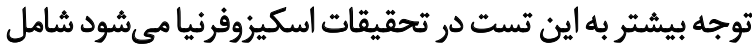

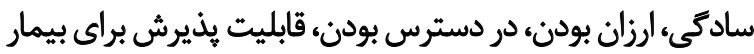
و بدون عارضه بودن آن است. يافتههاى مطالعه حاضر نشان داد كه در هر دو نقطه زمانى ني با كاهش غلظت محلول نياسين حساسيت تست يوان يوستى و ونيز

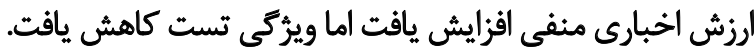

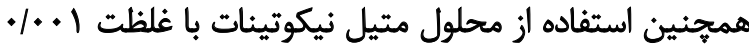

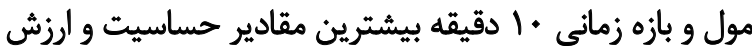

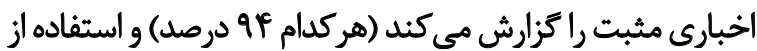

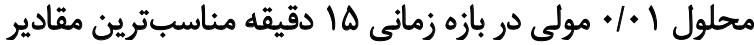

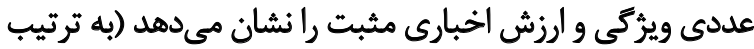

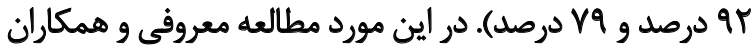

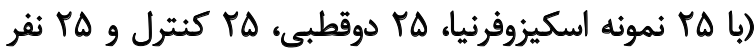

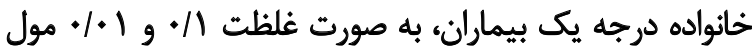

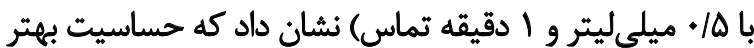

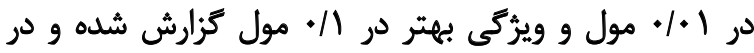

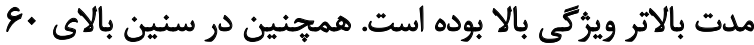

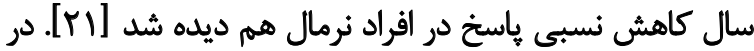

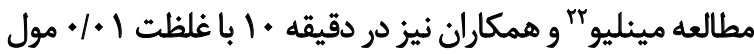

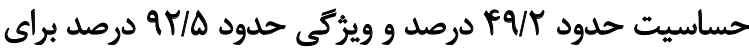

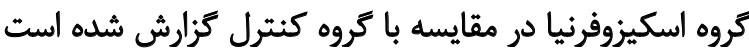

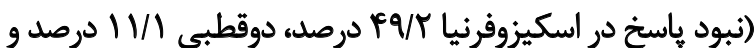

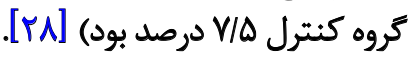
از يافتههاي مطالعه حاضر اين بود كه نتايج تست در هر دو دوريت

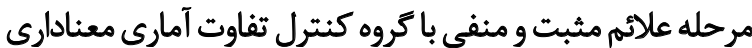

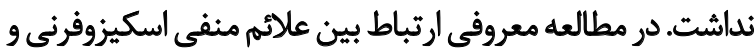

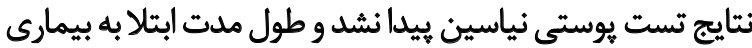

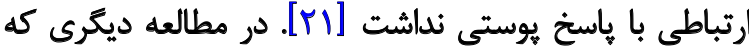

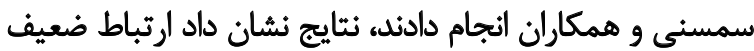

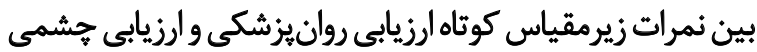

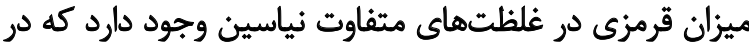

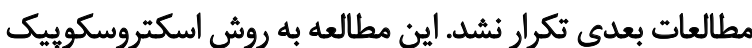

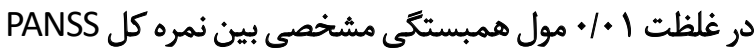

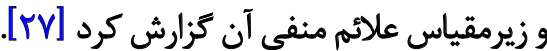

اين مطالعه با هدف ارزيابي ميزان صحت استفاده از بيوماركر

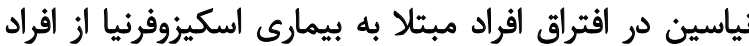

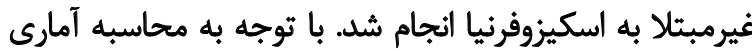

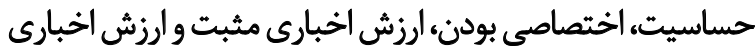

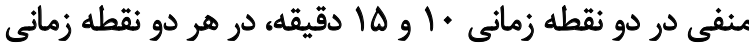

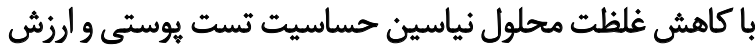

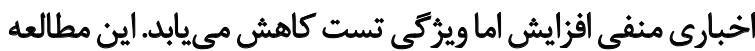

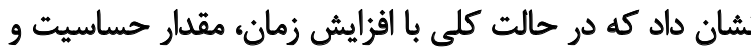

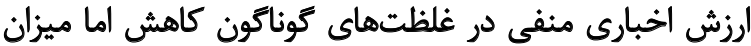

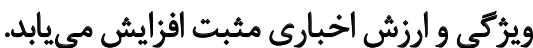

نتايج اين مطالعه منطبق با اكثر مطالعات صورت كرفته، بيان

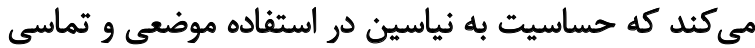

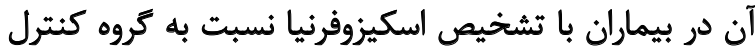

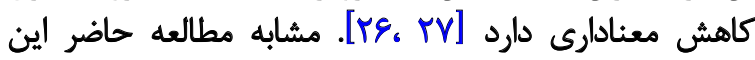

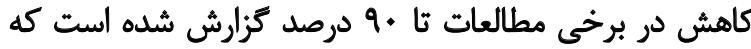

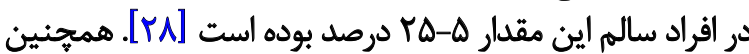

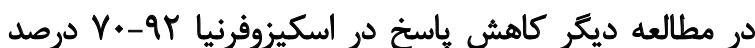

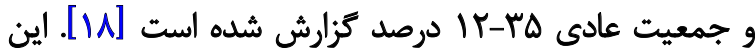

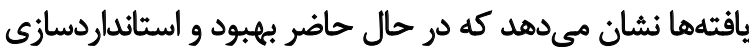

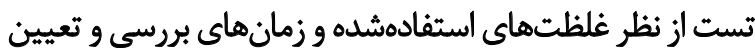

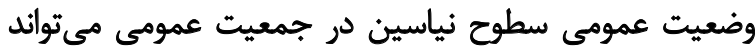

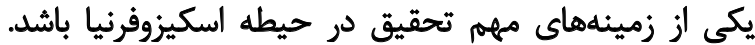

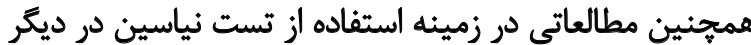

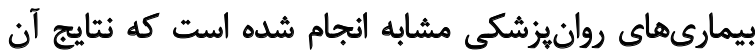

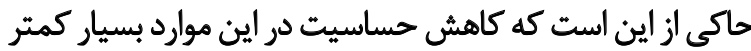

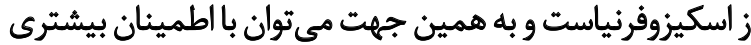

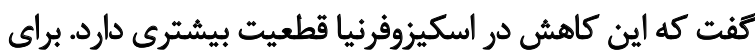

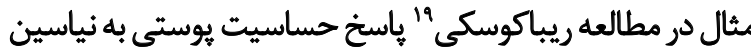

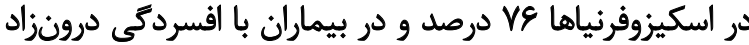

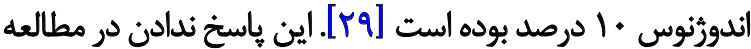

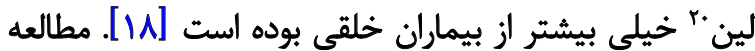

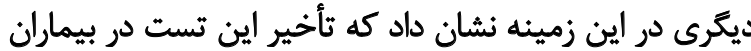

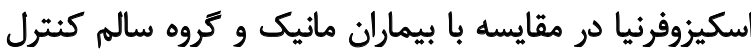

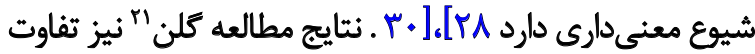

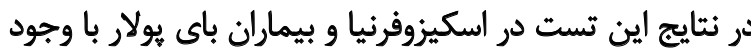

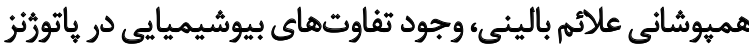
اين بيمارى را مطرح كرده است [استئين. در خصوص تضعيف ياخنثى شدن حساسيت يوستى در باسخ 
همه شركت كنندكان در جريان روند يُوهش بودند. اطلاعات آن

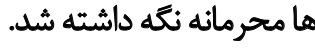

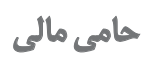

اين تحقيق هيج كونه كمك مالى ازٔ سازمان هاي تأمين مالى در بخش هاى عمومى ، تجارى يا غير انتفاعى دريافت نكردي

$$
\text { مشاركت نويسند مَّان }
$$

مفهومسازى، روششئاسى، اعتبارسنجى، تحليل: تمام

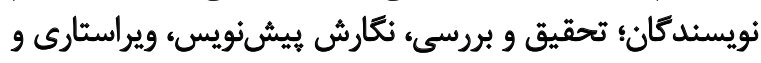

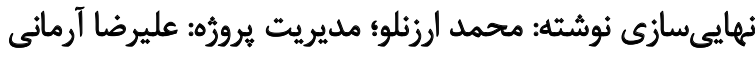
كيان.

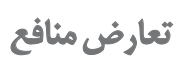

بنابر اظهار نويسندكان اين مقاله تعارض منافع ندارد.

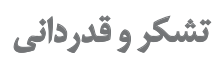

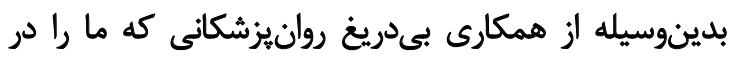

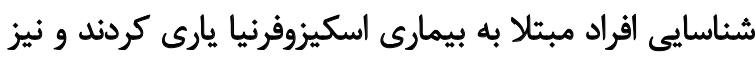

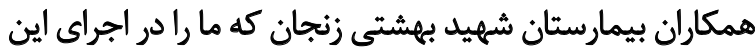

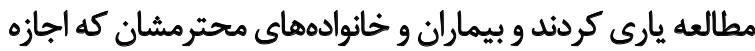
انجام تست در بدنشان را دادند قدردانى و سياسكزارى مي مكنيم.

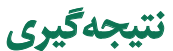

درمجموع نتايج اين مطالعه منطبق با اكثر مطالعات صورت

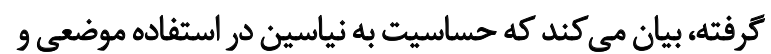

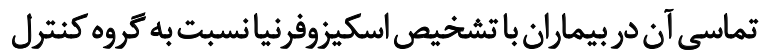

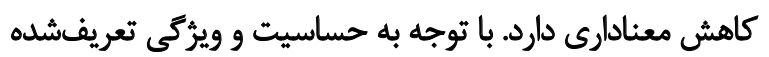

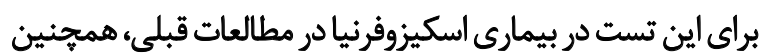

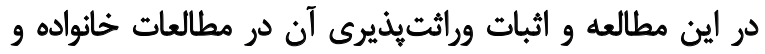

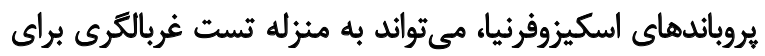

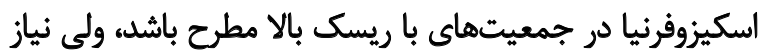

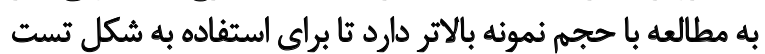
تشخيصى اطمينان آمارى مناسبى داشته باشد. اين مطالعه محدوديتهايى نيز دارد كه بايد در تفسير نتايج

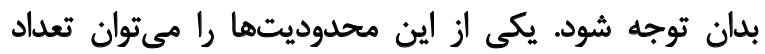

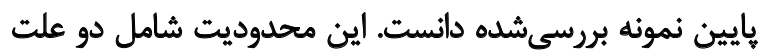

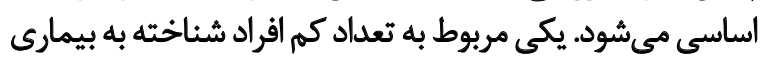

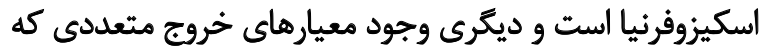

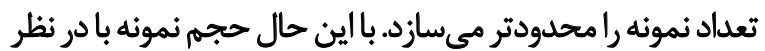

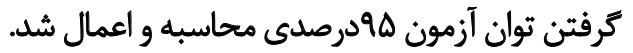

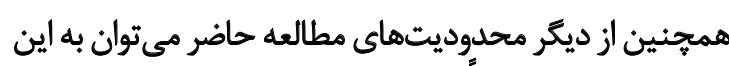

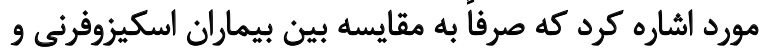

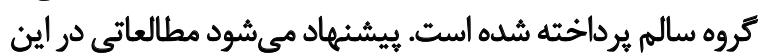

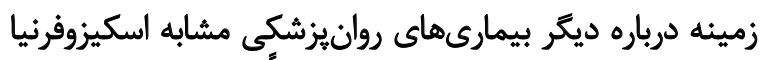

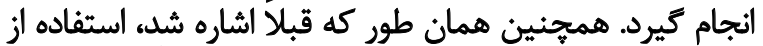

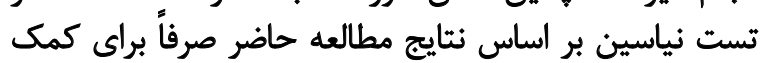

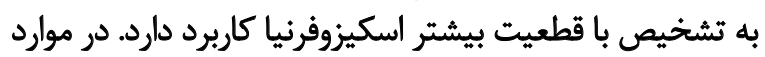

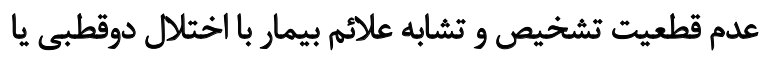

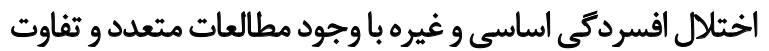

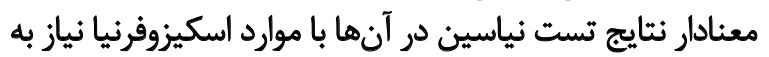
بررسى، تستهاى تشخيصى و مطالعات بيشترى است موارئ

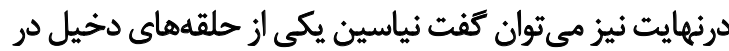

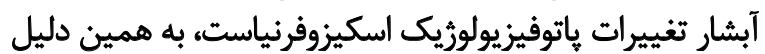

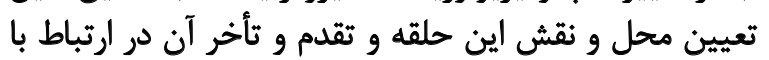

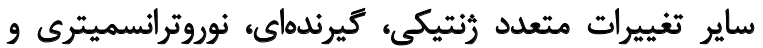

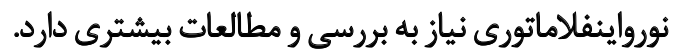

مالاحظاث اخلاقي ت مري

\section{يبيروى ازٔ اصول اخلاق يثوهش}

اين مقاله در كميته اخلاق دانشكاه علوميزشكى زنجان تصويب

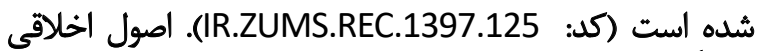

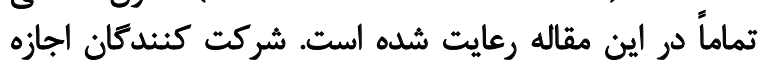

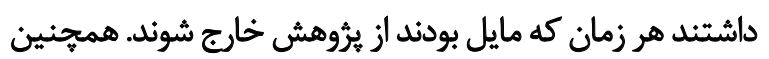




\section{References}

[1] Eaton WW, Martins SS, Nestadt G, Bienvenu OJ, Clarke D, Alexandre P. The burden of mental disorders. Epidemiologic Reviews. 2008; 30(1):1-14. [DOI:10.1093/epirev/mxn011] [PMID] [PMCID]

[2] Keshavan MS. Development, disease and degeneration in schizophrenia: A unitary pathophysiological model. Journal of Psychiatric Research. 1999; 33(6):513-21. [DOI:10.1016/S00223956(99)00033-3]

[3] Sadock BJ, Sadock VA, Ruiz P. Kaplan and Sadock's Comprehensive Textbook of Psychiatry. Philadelphia: Wolters Kluwer; 2017.

[4] Sadock BJ, Sadock VA, MD PR. Kaplan and Sadock's synopsis of psychiatry: Behavioral sciences/clinical psychiatry. Philadelphia: Wolters Kluwer; 2014

[5] Pinkham AE, Badcock JC. Assessing cognition and social cognition in schizophrenia \& related disorders. A clinical introduction to psychosis. Amsterdam: Elsevier; 2020. [DOI:10.1016/B978-012-815012-2.00008-0]

[6] McCutcheon RA, Reis Marques T, Howes OD. Schizophrenia-an Overview. JAMA Psychiatry. 2020; 77(2):201-10. [DOI:10.1001/ jamapsychiatry.2019.3360] [PMID]

[7] Jahanian AA, Rezaei O, Fadai F, Yaraghchi A. [The effectiveness of revisiting in reducing tardive dyskinesia symptoms in patients with Schizophrenia (Persian)]. Iranian Journal of Psychiatry and Clinical Psychology. 2014; 20(1):29-34. http://ijpcp.iums.ac.ir/ article-1-2143-en.html

[8] Talaei Zadeh S, Panaghi L, Hosseini SMM. The study of self-narration of patients with schizophrenia based on Genette's structural analysis theory. Iranian Journal of Psychiatry and Clinical Psychology. 2017; 23(3):336-47. [DOI:10.29252/nirp.ijpcp.23.3.336]

[9] Khodadadi N, Sheikholeslami F, Mahmudi H, Baghaie M, Hafezi M. [Comparison of quality of life of schizophrenic patients and healthy people in Rasht (Persian)]. Journal of Holistic Nursing And Midwifery. 2009; 19(61):8-15. https://hnmj.gums.ac.ir/ article-1-281-en.pdf

[10] Nuckols CC, Nuckols CC: The diagnostic and statistical manual of mental disorders, (DSM-5). Philadelphia: American Psychiatric Association 2013

[11] McGlashan TH. Duration of untreated psychosis in first-episode schizophrenia: Marker or determinant of course? Biol Psychiatry. 1999; 46(7):899-907. [DOI:10.1016/S0006-3223(99)00084-0]

[12] Çakici N, Van Beveren N, Judge-Hundal G, Koola M, Sommer I. An update on the efficacy of anti-inflammatory agents for patients with schizophrenia: A meta-analysis. Psychological Medicine. 2019; 49(14):2307-19. [DOI:10.1017/S0033291719001995] [PMID] [PMCID]

[13] McGorry PD, Goldstone SD, Parker AG, Rickwood DJ, Hickie IB. Cultures for mental health care of young people: An Australian blueprint for reform. The Lancet Psychiatry. 2014; 1(7):559-68. [DOI:10.1016/S2215-0366(14)00082-0]

[14] Group BDW, Atkinson Jr AJ, Colburn WA, DeGruttola VG, DeMets DL, Downing GJ, et al. Biomarkers and surrogate endpoints: Preferred definitions and conceptual framework. Clinical Pharmacology \& Therapeutics. 2001; 69(3):89-95. [DOI:10.1067/ mcp.2001.113989] [PMID]
[15] Berger GE, Smesny S, Schäfer MR, Milleit B, Langbein K, Hipler UC, et al. Niacin skin sensitivity is increased in adolescents at ultra-high risk for psychosis. PloS One. 2016; 11(2):e0148429. [DOI:10.1371/journal.pone.0148429] [PMID] [PMCID]

[16] Chang SS, Liu CM, Lin SH, Hwu HG, Hwang TJ, Liu SK, et al. Impaired flush response to niacin skin patch among schizophrenia patients and their nonpsychotic relatives: the effect of genetic loading. Schizophrenia Bulletin. 2008; 35(1):213-21. [DOI:10.1093/ schbul/sbm153] [PMID] [PMCID]

[17] Lien YJ, Huang SS, Liu CM, Hwu HG, Faraone SV, Tsuang MT, et al. A genome-wide quantitative linkage scan of niacin skin flush response in families with schizophrenia. Schizophrenia Bulletin. 2011; 39(1):68-76. [DOI:10.1093/schbul/sbr054] [PMID] [PMCID]

[18] Lin SH, Liu CM, Chang SS, Hwu HG, Liu SK, Hwang TJ, et al Familial aggregation in skin flush response to niacin patch among schizophrenic patients and their nonpsychotic relatives. Schizophrenia Bulletin. 2007; 33(1):174-82. [DOI:10.1093/schbul/sbl038] [PMID] [PMCID]

[19] Horrobin D. Schizophrenia as a prostaglandin deficiency disease. The Lancet. 1977; 309(8018):936-7. [DOI:10.1016/S01406736(77)92228-0]

[20] Nadalin S, Buretić-Tomljanović A, Rubeša G, Tomljanović D, Gudelj L. Niacin skin flush test: A research tool for studying schizophrenia. Psychiatria Danubina. 2010; 22(1):14-27. https:// hrcak.srce.hr/48604

[21] Maroufi M, Tabatabaeian M, Tabatabaeian M, Mahaki B, Teimoori G. Comparison of niacin skin flush response in patients with schizophrenia and bipolar disorder. Iranian Journal of Psychiatry and Behavioural Sciences. 2016; 10(4):e504 [DOI:10.17795/ijpbs-504]

[22] Kay SR, Fiszbein A, Opler LA. The Positive and Negative Syndrome Scale (PANSS) for Schizophrenia. Schizophrenia Bulletin. 1987; 13(2):261-76. [DOI:10.1093/schbul/13.2.261] [PMID]

[23] Jackson C. The general health questionnaire. Occupational Medicine. 2007; 57(1):79. [DOI:10.1093/occmed/kql169]

[24] Ward P, Sutherland J, Glen E, Glen A. Niacin skin flush in schizophrenia: A preliminary report. Schizophrenia Research. 1998; 29(3):269-74. [DOI:10.1016/S0920-9964(97)00100-X]

[25] Yaseri M, Pakpour A, Rahmani S, Rangin H, Akaberi A. SelfLearning concepts of diagnostic tests by graphical approach: sensitivity, specificity, positive predictive value and negative predictive value. Journal of North Khorasan University of Medical Sciences. 2012; 4(2):275-82. [DOI:10.29252/jnkums.4.2.275]

[26] Allen AJ, Griss ME, Folley BS, Hawkins KA, Pearlson GD. Endophenotypes in Schizophrenia: A selective review. Schizophrenia Research. 2009; 109(1-3):24-37. [DOI:10.1016/j.schres.2009.01.016] [PMID] [PMCID]

[27] Smesny S, Klemm S, Stockebrand M, Grunwald S, Gerhard U-J, Rosburg T, et al. Endophenotype properties of niacin sensitivity as marker of impaired prostaglandin signalling in schizophrenia. Prostaglandins, Leukotrienes and Essential Fatty Acids. 2007 77(2):79-85. [DOI:10.1016/j.plefa.2007.08.006] [PMID]

[28] Hudson CJ, Lin A, Cogan S, Cashman F, Warsh JJ. The niacin challenge test: clinical manifestation of altered transmembrane signal transduction in schizophrenia? Biological Psychiatry. 1997; 41(5):507-13. [DOI:10.1016/S0006-3223(96)00112-6] 
[29] Rybakowski J, Weterle R. Niacin test in Schizophrenia and affective illness. Biological psychiatry. 1991; 29(8):834-6. [DOI:10.1016/0006-3223(91)90202-W]

[30] Liu CM, Chang SS, Liao SC, Hwang TJ, Shieh MH, Liu SK, et al. Absent response to niacin skin patch is specific to schizophrenia and independent of smoking. Psychiatry Research. 2007; 152(23):181-7. [DOI:10.1016/j.psychres.2006.10.002] [PMID]

[31] Glen A, Cooper S, Rybakowski J, Vaddadi K, Brayshaw N, Horrobin $\mathrm{D}$. Membrane fatty acids, niacin flushing and clinical parameters. Prostaglandins, Leukotrienes and Essential Fatty Acids. 1996; 55(1-2):9-15. [DOI:10.1016/S0952-3278(96)90139-8]

[32] Tavares Jr H, Yacubian J, Talib LL, Barbosa NR, Gattaz WF. Increased phospholipase A2 activity in schizophrenia with absent response to niacin. Schizophrenia Research. 2003; 61(1):1-6. [DOI:10.1016/S0920-9964(02)00281-5]

[33] Sadock BJ, Sadock VA. Kaplan \& Sadock's concise textbook of clinical psychiatry. Philadelphia: Lippincott Williams \& Wilkins; 2008.

[34] Puri B, Easton T, Das I, Kidane L, Richardson A. The niacin skin flush test in schizophrenia: A replication study. International Journal of Clinical Practice. 2001; 55(6):368-70. [PMID]

[35] Horrobin DF. Schizophrenia as a membrane lipid disorder which is expressed throughout the body. Prostaglandins, Leukotrienes and Essential Fatty Acids. 1996; 55(1-2):3-7. [DOI:10.1016/ S0952-3278(96)90138-6]

[36] McClellan JM, Susser E, King MC. Schizophrenia: A common disease caused by multiple rare alleles. The British Journal of Psychiatry. 2007; 190(3):194-9. [DOI:10.1192/bjp.bp.106.025585] [PMID]

[37] Puri BK, Hirsch SR, Easton T, Richardson AJ. A volumetric biochemical niacin flush-based index that noninvasively detects fatty acid deficiency in schizophrenia. Progress in Neuro-Psychopharmacology and Biological Psychiatry. 2002; 26(1):49-52. [DOI:10.1016/S0278-5846(01)00220-2] 